\title{
Single Dose Methylphenidate Does Not Impact on Attention and Decision Making in Healthy Medical Students
}

\author{
Leah Shalev a, b, Varda Gross-Tsur ${ }^{\mathrm{a}}$, Yehudah Pollak
}

\begin{abstract}
Background: Methylphenidate (MPH) is effective for the treatment of Attention Deficit Hyperactivity Disorder (ADHD) and used illicitly by healthy adults, even though evidence concerning its efficacy is inconsistent and equivocal. We studied the effect of MPH on two cognitive tasks (attention/inhibition and decision making), a subjective rating scale and heart rate and blood pressure.
\end{abstract}

Methods: Forty five medical students, ages 20 - 30, who denied past or present ADHD symptoms, participated in this double-blind, randomized, placebo controlled cross-over experiment. Data collection was conducted in two sessions, each 2.5 hours, two weeks apart. At the beginning of each session, the subjects completed a Visual Analogue Scale (VAS) quantifying their feelings regarding present mental and emotional state and then administered either placebo or MPH $(0.3 \mathrm{mg} / \mathrm{kg})$. Ninety minutes later, they again completed the VAS and were administered two cognitive tasks: Test of Variables of Attention (TOVA) and the modified Cambridge Gambling Test (mCGT). We found no differences with or without MPH for reaction time, response time variability, number of commissions and omissions in the TOVA or for quality, sum of gamble and reaction time for the mCGT.

Results: No differences were observed between sessions with or without methylphenidate for reaction time, response time variability, number of commissions and omissions in the TOVA or for quality, sum of gamble and reaction time for the mCGT. Furthermore, no differences were observed in subjective rating on the VAS. Small, non-significant increases in blood pressure were documented. A practice effect was noted for the two consecutive sessions regardless of intervention.

Manuscript accepted for publication October 1, 2012

\footnotetext{
${ }^{\mathrm{a}}$ Neuropediatric Unit, Shaare Zedek Medical Center, POB 3235 , Jerusalem, 91031, Israel

${ }^{\mathrm{b}}$ Corresponding author: Leah Shalev, Neuropediatric Unit, Shaare Zedek

Medical Center, POB 3235, Jerusalem, 91031, Israel.

Email: shalevleah@gmail.com
}

doi: http://dx.doi.org/10.4021/jnr143w
Conclusions: In this study, MPH did not affect sustained attention and decision making in healthy young adults, did not alter the subjective perception of their mental and emotional state nor influence heart rate or blood pressure.

Keywords: Methylphenidate; Attention; Decision making; Behavioral assessment

\section{Introduction}

Methylphenidate is a psychostimulant used to treat ADHD, reducing the symptoms of inattention, hyperactivity and impulsivity in over $70 \%$ of cases [1]. A steep increase in the use of psychostimulants has been documented since the 1980's and prescription records indicate that the swell can be attributed to individuals 15 years and older [2]. This increase may reflect correction of unrecognized ADHD in adolescents and young adults. However, during this same time period, psychostimulants have been diverted to non-clinical use as cognitive enhancers. A series of surveys found that $8 \%$ of middle, high school and college students used psychostimulants to improve cognitive function [3] while an informal survey conducted by the scientific journal NATURE among 1,600 of its readers revealed that $20 \%$ had taken a non-prescribed psychostimulant to improve concentration [4]. The most common psychostimulant reported in this survey was MPH $(62 \%)$. Another survey of 4,580 undergraduates found that $8.3 \%$ used a psychostimulant during the previous year and another $6 \%$ during the course of their lifetime to improve concentration, aid in study and increase alertness [5]. In spite of the widespread use of MPH as a cognitive enhancer, multiple cognitive tasks have not unequivocally demonstrated its efficacy in healthy adults. For example, although MPH enhanced vigilance in two studies [6,7], other research found this effect only under special conditions such as task novelty [8] or its challenging nature [9]. Conflicting data was found for the effect of MPH under conditions of sleep deprivation, enhancing vigilance in nine sleep deprived individuals [10] but ineffective in 20 other carefully selected students whether sleep deprived or not [11]. 
Table 1. The Average Values for Each Phase of the TOVA. Phase 1 and 2 are the "Target Frequent" Phases and Phase 3 and 4 are the "Target Infrequent" Phases Which Explains the Sharp Increase in Number of Commissions

\begin{tabular}{|c|c|c|c|c|c|}
\hline & & \multicolumn{4}{|c|}{ Phase $($ mean \pm std $)$} \\
\hline & & 1 & 2 & 3 & 4 \\
\hline \multirow[t]{5}{*}{ Placebo } & Omissions (\%) & $0.6 \pm 0.41$ & $0.49 \pm 1.48$ & $0.39 \pm 1.31$ & $0.37 \pm 1.47$ \\
\hline & Commissions (\%) & $0.32 \pm 0.61$ & $0.23 \pm 0.40$ & $10.02 \pm 8.92$ & $10.80 \pm 10.54$ \\
\hline & RT (msec) & $340 \pm 45$ & $348 \pm 47$ & $301 \pm 46$ & $305 \pm 52$ \\
\hline & RT variability (msec) & $53 \pm 17$ & $54 \pm 16$ & $64 \pm 21$ & $69 \pm 27$ \\
\hline & D prime & $7.95 \pm 0.92$ & $7.71 \pm 1.31$ & $5.71 \pm 1.46$ & $5.47 \pm 1.27$ \\
\hline \multirow[t]{5}{*}{$\mathrm{MPH}$} & Omsissions (\%) & $0.00 \pm 0.00$ & $0.06 \pm 0.41$ & $0.10 \pm 0.32$ & $0.19 \pm 0.97$ \\
\hline & Commissions $(\%)$ & $0.38 \pm 0.60$ & $0.28 \pm 0.45$ & $8.64 \pm 7.40$ & $10.30 \pm 9.59$ \\
\hline & $\mathrm{RT}(\mathrm{msec})$ & $334 \pm 41$ & $344 \pm 45$ & $299 \pm 40$ & $297 \pm 49$ \\
\hline & RT variability (msec) & $51 \pm 14$ & $55 \pm 26$ & $60 \pm 22$ & $62 \pm 20$ \\
\hline & D prime & $7.83 \pm 0.94$ & $7.89 \pm 0.91$ & $5.82 \pm 1.19$ & $5.76 \pm 1.21$ \\
\hline
\end{tabular}

Using a MANOVA test, no significant difference was found between placebo and MPH $(n=45, P>0.05)$.

While the results of cognitive tasks used to assess classical components of attention and executive functions are equivocal, the effect of MPH on probabilistic decision making is even less clear. Decision making is a motivation driven, risk taking process assessed using gambling paradigms. The influence of MPH on decision making has been assessed in several pathologies. Children with ADHD [12] (but not adults with ADHD [13]) and adults with fronto-temporal dementia [14] decrease the sum of bet on a gambling test, reflecting attenuation of risk taking. However, there was no influence of the drug on the quality of the choice or on the response time, variables that are usually attributed to the drug's known inhibitory effect, suggesting that the drug may have yet another, unknown mechanism of action in the process of decision making.

Alternatively, healthy individuals may choose to use this drug because of its subjective effect on perception of self confidence, [11] sense of concentration, [15] alertness and vigor, fatigue $[6,8]$, mood $[6]$ and well being $[9,16,17]$.

Given the range of possible effects of MPH, there are several explanations for its non-prescription use among healthy adults. It may improve cognitive function in healthy individuals, elicit subjective feelings suitable for accomplishing cognitive tasks or augment the autonomic sympathetic system subconsciously preparing for stressful or challenging assignments. Other options include an anticipatory placebo effect or self treatment in undiagnosed individuals with ADHD. To test the effects of MPH on attention and decision making in healthy young adults and discriminate between these possibilities, we studied a large homogenous group of medical students in whom ADHD and other related disorders were carefully ruled out. The experimental paradigm included cognitive functions, subjective rating and physiological measures of heart rate and blood pressure.

\section{Methods}

A within-subject, crossover, double-blind, placebo-control design was employed in this study. Fifty three medical students responded to the research advertisements. Each volunteer was contacted by telephone in order to determine if the subject had or has a diagnosis of ADHD or any other learning disability; current or former use of MPH or illicit drugs (cocaine, amphetamines, heroin), sudden death in first or second degree relatives; signs or symptoms of structural heart disease, psychiatric or medical conditions, pregnancy or chronic medical treatment. Only if the answer to all of the questions was negative did the subjects proceed to the second screening stage. This consisted of three electronic questionnaires that were sent via email to 50 volunteers prior to the first meeting. These standardized questionnaires screen for current symptoms of ADHD (Adult ADHD Self Report Scale [18], ASRS), childhood ADHD (Wender Utah Rating 
Table 2. The Average Values for Each Ratio on the mCGT

\begin{tabular}{llllll}
\hline & & \multicolumn{2}{c}{ Ratio (mean \pm std) } \\
\cline { 3 - 5 } & & $\mathbf{1 : 9}$ & $\mathbf{2 : 8}$ & $\mathbf{3 : 7}$ \\
\hline Placebo & RT (msec) & $1,272 \pm 994$ & $1,331 \pm 878$ & $1.433 \pm 926$ & $1.584 \pm 780$ \\
& Sum of bet (\$) & $88 \pm 13$ & $78 \pm 16$ & $69 \pm 17$ & $55 \pm 21$ \\
MPH & Quality of gamble (\%) & $99.25 \pm 3.47$ & $98.51 \pm 5.96$ & $99.25 \pm 3.47$ & $94.44 \pm 12.30$ \\
& RT (msec) & $1,153 \pm 567$ & $1,198 \pm 540$ & $1.424 \pm 832$ & $1.554 \pm 862$ \\
& Sum of bet (\$) & $89 \pm 14$ & $79 \pm 16$ & $67 \pm 17$ & $52 \pm 20$ \\
\hline
\end{tabular}

Using a MANOVA test, no significant difference was found between placebo and MPH $(n=45, P>0.05)$.

Scale, [19] WURS) and other psychiatric conditions (Brief Symptom Inventory, [20] BSI). We used the cutoff scores suggested by the authors for the ASRS and WURS: less than four of six shaded squares for the first part of the ASRS, less than 36 points on the WURS and one or more standard deviation above the Israel norms as the cutoff for the BSI. Subjects with a single score outside the range were excluded. 45 volunteers ( 20 women and 25 men) were found eligible.

Participants were then scheduled for two, 2.5 hour-meetings, in the Neuropediatric Unit of the Shaare Zedek Medical Center, Jerusalem. Subjects were asked to refrain from alcohol (24 hours) and caffeine (12 hours) before the experimental sessions. A minimum of one week was assigned between the two sessions. Heart rate (HR) and blood pressure (BP) were obtained (the average of 3 consecutive measures using calibrated electronic equipment) at the beginning of each meeting. Women were asked again about possible pregnancy and underwent a urine sample test. In addition, each subject completed a visual analogue scale (VAS), a measure of subjective perceptions, composed of 16 questions. Then, in a randomized, double blind fashion, the subject received a capsule of either MPH or placebo. Ninety minutes [21] later, HR and BP measurements were obtained, the VAS was completed again and the subjects were administered two cognitive tasks. The study was approved by the hospital internal review board and on the day of the first meeting each participant signed an informed consent. Each participant was monetarily compensated at the end of the second session.

\section{Pharmacological treatment}

Ninety minutes preceding testing, subjects received a capsule containing either MPH (15 - $20 \mathrm{mg}$, approximately corresponding to $0.3 \mathrm{mg} / \mathrm{kg} \mathrm{MPH}$ ) or placebo. The order of administration (MPH or placebo) was determined by ran- dom assignment, unknown both to the participant and the research assistant.

Tools

Test of Variables of Attention (TOVA): The TOVA incorporates a standardized two second inter-stimulus interval during a 21.6 min test [22]. The paradigms are target infrequent and target frequent. In the first part of the test, a 3.5:1 ratio of non-targets to targets is presented while in the second part the ratio is reversed. The participant is instructed to press the micro switch as quickly and accurately as possible when the target appears on the computer screen. Five measures are derived from the TOVA: response time, response time variability, omissions (not responding to a target), and commissions (responding to a non target), and d' prime, automatically calculated by the computer software for each quarter (phase) of the test.

Modified Cambridge Gambling Task (mCGT): The participants were told that the computer has randomly hidden a yellow token inside one of ten red or blue boxes appearing on the screen [23]. The subject first guesses whether the token is hidden under a red or blue box and then decides upon how many points to gamble. The subject is presumed to understand that the likelihood of each choice to be correct is a function of the ratio of red to blue boxes displayed. This produces a range of situations from more likely (9:1) to less likely (6:4). Immediately after choosing, a message and sound appear signifying the results of the bet (Win/Lose). If the subject chooses the correct color, the bet placed is added to his score; if wrong, the bet is subtracted. The subject is instructed to treat the points as being valuable and to accumulate as many as possible.

This modified CGT (mCGT) version differs from the original in that the gamble options are equal at all rounds 
Table 3. The Average of Differences (After - Before) Between Cardiovascular Measures in Both Experiment Conditions

\begin{tabular}{lllll}
\hline & \multicolumn{2}{c}{ Placebo } & \multicolumn{2}{c}{ MPH } \\
\cline { 2 - 4 } & Mean & Std. deviation & Mean & Std. deviation \\
\hline HR (bpm) & -6.88 & 10.13 & -4.87 & 7.40 \\
Systolic BP (mmHg) & -0.15 & 9.00 & 2.86 & 8.83 \\
Diastolic BP (mmHg) & 0.44 & 6.99 & 4.08 & 7.25 \\
\hline
\end{tabular}

Using a MANOVA test, no measure reached significant difference. A negative value signifies a decrease on second measurement. ( $n=45, P>0.05)$ (bpm: beats per minute; BP: blood pressure).

regardless of the so-far accumulated points, so that subjects consider each session in terms of its immediate net outcome and the available bets appear all at once to eliminate responses based on motor impulsivity or a delay aversion.

Three measures of DM were used: (1) sum of bet (points staked on trials in which a probability-correct choice was made); (2) quality of decision (percentage of probabilitycorrect trials chosen) and (3) reaction time (time until color is chosen).

\section{Results}

We were unable to demonstrate that MPH altered or enhanced performance on either the TOVA or the mCGT (Table 1,2).

\section{TOVA}

Using MANOVA with repeated measures, no drug effect for the TOVA was found, nor were effects for each of its variables when analyzed with ANOVA. To assess the role of practice and possible interactions between practice and treatment, we compared the first and second sessions, regardless of the order in which subjects received drug or placebo. This comparison revealed practice effects for response time variability $(\mathrm{P}<0.005)$, number of commissions $(\mathrm{P}<0.001)$ and d' prime $(P<0.01)$. Importantly, no treatment effect was found when comparing placebo versus MPH on the first session in a between-subject analysis.

\section{mCGT}

For the mCGT, MANOVA with repeated measures revealed no effect for the drug nor did ANOVA for each of the mCGT variables. A large effect was found for the ratio $(\mathrm{P}<0.001)$. Specifically, three changes were noted: as the ratio between red and blue boxes increased (significantly more of one color), the sum of bet increased ( $\mathrm{P}<0.001)$, the quality of gamble improved $(\mathrm{P}<0.01)$ and response time shortened $(\mathrm{P}$ $<0.001)$. A treatment effect was found on several of the measures however this effect failed to reach significance once the session order was co-varied. To assess the role of practice and possible interactions between practice and treatment, the comparison between first and second sessions revealed practice effects for sum of bet $(\mathrm{P}<0.005)$ and response time $(\mathrm{P}<$ 0.001 ), regardless of the order of intervention. Importantly, no treatment effect was found when comparing placebo versus MPH on the first session in a between-subject analysis.

\section{Physiologic measures:}

Heart rate, systolic and diastolic blood pressure were calculated as the average of three values obtained consecutively before and 90 minutes after treatment. A decrease in heart rate ( $7 \mathrm{bpm}$ for the placebo arm, $5 \mathrm{bpm}$ for the MPH arm), an increase of $3 \mathrm{mmHg}$ in systolic blood pressure (-0.15 in the placebo session, $3 \mathrm{mmHg}$ in the MPH session) and an increase of $4 \mathrm{mmHg}$ in diastolic blood pressure $(0.4 \mathrm{mmHg}$ for the placebo session, $4 \mathrm{mmHg}$ for the MPH session) were documented (Table 3).

Though no significance using MANOVA with repeated measures could be demonstrated, we observed a trend in which MPH curbed the decrease in heart rate and increased blood pressure which could not be attributed to the order of sessions.

\section{Visual analogue scale:}

The participants' subjective perceptions (Table 4) revealed no drug effect.

\section{Discussion}

In this study, we did not document a cognitive enhancing 
Table 4. The Average of Differences (After - Before) Between Subjective Measures in Both Experiment Conditions

\begin{tabular}{|c|c|c|c|c|}
\hline & \multicolumn{2}{|c|}{ Placebo } & \multicolumn{2}{|c|}{ МPH } \\
\hline & Mean (mm) & Std. deviation & Mean (mm) & Std. deviation \\
\hline Friendly - Withdrawn & -0.77 & 11.72 & 1.25 & 9.90 \\
\hline Alert - Drowsy & 0.05 & 22.4 & -6.50 & 20.08 \\
\hline Calm - Excited & -5.27 & 17.68 & -1.64 & 18.52 \\
\hline Clear minded - Fuzzy & 6.25 & 14.41 & 4.82 & 17.06 \\
\hline Coordinated - Clumsy & 3.36 & 11.26 & 3.89 & 14.03 \\
\hline Energetic - Lethargic & 4.09 & 19.11 & -1.68 & 17.82 \\
\hline Troubled - Tranquil & -0.64 & 14.39 & -2.95 & 16.72 \\
\hline Contented - Discontented & 1.50 & 10.75 & -5.30 & 12.73 \\
\hline Strong - Feeble & 3.05 & 14.59 & 1.68 & 16.19 \\
\hline Mentally slow - Quick witted & -2.18 & 15.17 & -1.20 & 12.06 \\
\hline Tense - Relaxed & -1.11 & 15.30 & -3.30 & 12.23 \\
\hline Attentive - Dreamy & 0.41 & 14.38 & 1.30 & 12.21 \\
\hline Proficient - Incompetent & 1.66 & 8.40 & 0.41 & 11.47 \\
\hline Happy - Sad & -2.05 & 10.29 & -2.45 & 12.28 \\
\hline Amicable - Antagonistic & 0.43 & 8.50 & 0.02 & 7.69 \\
\hline Interested - Bored & 1.02 & 13.06 & -2.36 & 11.24 \\
\hline
\end{tabular}

Using a T-test, no significant difference was found for any pair. A negative value signifies a tendency towards the right handed adjective. $(n=45, P>0.05)$.

effect of MPH on normal, healthy, high achieving students, a group at risk for use of non-prescription psychostimulants. Furthermore, our subjects also did not report subjective changes in vigor, alertness, interest, attentiveness or motivation. However, we did find a learning effect as evident by the improvement in the second test sessions compared to the first, regardless of medication state.

A possible explanation for the lack of effect is the rigorous two-stage screening that the subjects underwent to rule out undiagnosed ADHD. Volunteers who answered positively on the initial screening telephone interview to questions regarding a previous diagnosis of ADHD, learning disability, other psychiatric, behavioral or systemic disorders or previous use of psychostimulants or any other chronic medical treatment were excluded a priori. In the second screening, subjects completed multiple questionnaires to identify these disorders and low-threshold clinical cutoff criteria were used. Thus, it is unlikely that undiagnosed individuals were included in the group, a phenomenon that may have contributed to positive results in other studies where less rigorous criteria were used $[10,13]$. In addition, the subjects received detailed instructions regarding each stage of the test to be certain that their performance would be optimal. It is unlikely that the medication dose was inappropriate since the physiological data (blood pressure and heart rate) were consistent with MPH effects on the sympathetic system in other studies with positive cognitive results $[6,8]$.

Although the TOVA has proved useful for the assessment of psychostimulant efficacy in ADHD population [24, 25], in our normal population, MPH had no impact on the different parameters of the TOVA. This finding is at odds with previous studies $[6,8]$, examining the effect of MPH 
on a non-TOVA CPT showing that MPH increased precision and shortened response time. One might expect that some of these inconsistencies could be explained by differences in the characteristics of the CPTs. However, it is difficult to attribute the differences in results to the length of the CPT since both a short (6 minute) and long (45 minute) CPT were used with similar results albeit different from the results of the 22 minute TOVA. Furthermore, some researchers have argued that the effect of MPH becomes apparent only when multiple cognitive functions are simultaneously challenged [26]. This interpretation may explain the inconsistencies between our results and those previously published because the TOVA, by definition, taxes only attention and response inhibition whereas the non-TOVA CPTs used require working memory as well. On the other hand, our results in healthy young adults are in line with a recent study of the effect of $\mathrm{MPH}$ in normal young adults for whom MPH had no effect on the TOVA. However, these authors also found the same result for their subjects with ADHD [13], a finding inconsistent with the bulk of literature confirming that the TOVA is sensitive to MPH in individuals with ADHD [24, 25].

Another potential masking factor could be the relatively high level of intelligence in our population, which is considered by the creators of the TOVA to have an impact on the results. However, this conclusion largely applies to children and adolescents, whereas in young adult population there is apparently no correlation between IQ and TOVA results [27]. Furthermore, it is not possible to claim that a ceiling effect masked any medication-induced changes since the observed change in the second session implied an ability to improve. Therefore our findings lead us to conclude that MPH does not affect attention in healthy young adults, as measured by TOVA.

To examine the effect of MPH on decision making we used the mCGT. Our hypothesis, that MPH would decrease the sum of bet, reflecting a stronger ability to employ cautiousness and improve the quality of gamble, was not supported by the results. We hypothesized that the risk taking component of the gambling task is age dependant so that young adults (17 - 27 years) tend, more than other groups, to risk larger sums while also adjusting the sum of bet according to the changing probability [28]. This pattern was found not true for children, especially not for those with ADHD. In children with ADHD the quality of gamble, risk adjustment and sum of bet are of lower quality than healthy controls [12] and treatment with MPH positively affected sum of bet. In our young adults, a group of an age where there is a strong tendency towards risk taking, MPH did not demonstrate a similar effect on sum of bet. On the contrary, regardless of medication state, subjects increased the sum of bet from the first to second session indicating that MPH did not have an impact on a possible learning effect.

Limitations of study: The cognitive tasks used have been designed to isolate a specific cognitive function and enable its measurement, but do not necessarily recapitulate real life situations for which individuals without ADHD take MPH. Second, the dose of MPH given to our subjects was derived from the clinical dose generally used for treating ADHD and from previous studies that did not demonstrate an advantage for a larger dose [29]. However, evidence of variable metabolism [21] may necessitate individual dose adjustment to achieve the optimal effect in healthy individuals. Finally, it may be that our population sample is not representative of the populations using cognitive enhancers that probably include individuals with undiagnosed ADHD or those with a tendency towards ADHD who report benefit from MPH [30].

In summary, in this randomized, placebo controlled within subject study of healthy medical students, MPH did not have an effect on the executive functions of attention and decision making nor did it alter their subjective feelings such as increased interest, attention or alertness. Given the lack of unequivocal data indicating that MPH indeed meaningfully alters cognitive functions in healthy young adults, it is not clear why this group chooses to use stimulants. Focused research on this phenomenon is necessary to establish the causes for the widespread use of MPH in healthy young adults.

\section{Acknowledgement}

We would like to thank all our participants who volunteered to take part in the study.

This work was supported by the neuropediatric unit of the Sharee Zedek Medical Center. The TOVA company provided gratis 50 of the TOVA tests.

\section{Conflict of Interests}

The authors declare that there is not conflict of interest.

\section{Registration Information}

Trial registry name - Methylphenidate in Healthy Young Adults, Registration identification number - NCT00815841. URL -https://register.clinicaltrials.gov/prs/app/action/Select Protocol?sid=S0001SQM\&selectaction=View\&uid $=\mathrm{U} 0000$ EFO\&ts $=10 \& \mathrm{cx}=$ oea 6 ea

\section{References}

1. Wilens TE. Effects of methylphenidate on the catecholaminergic system in attention-deficit/hyperactivity disorder. J Clin Psychopharmacol. 2008;28(3 Suppl 2):S46-53. 
2. Swanson JM, Volkow ND. Psychopharmacology: concepts and opinions about the use of stimulant medications. J Child Psychol Psychiatry. 2009;50(1-2):180193.

3. McCabe SE, Teter CJ, Boyd CJ, Guthrie SK. Prevalence and correlates of illicit methylphenidate use among 8th, 10th, and 12th grade students in the United States, 2001. J Adolesc Health. 2004;35(6):501-504.

4. Maher B. Poll results: look who's doping. Nature. 2008;452(7188):674-675.

5. Teter CJ, McCabe SE, LaGrange K, Cranford JA, Boyd CJ. Illicit use of specific prescription stimulants among college students: prevalence, motives, and routes of administration. Pharmacotherapy. 2006;26(10):15011510 .

6. Strauss J, Lewis JL, Klorman R, Peloquin LJ, Perlmutter RA, Salzman LF. Effects of methylphenidate on young adults' performance and event-related potentials in a vigilance and a paired-associates learning test. Psychophysiology. 1984;21(6):609-621.

7. Camp-Bruno JA, Herting RL. Cognitive effects of milacemide and methylphenidate in healthy young adults. Psychopharmacology (Berl). 1994;115(1-2):46-52.

8. Elliott R, Sahakian BJ, Matthews K, Bannerjea A, Rimmer J, Robbins TW. Effects of methylphenidate on spatial working memory and planning in healthy young adults. Psychopharmacology (Berl). 1997;131(2):196206.

9. Volkow ND, Wang GJ, Fowler JS, Telang F, Maynard L, Logan J, Gatley SJ, et al. Evidence that methylphenidate enhances the saliency of a mathematical task by increasing dopamine in the human brain. Am J Psychiatry. 2004;161(7):1173-1180.

10. Bishop C, Roehrs T, Rosenthal L, Roth T. Alerting effects of methylphenidate under basal and sleep-deprived conditions. Exp Clin Psychopharmacol. 1997;5(4):344352.

11. Bray CL, Cahill KS, Oshier JT, Peden CS, Theriaque DW, Flotte TR, Stacpoole PW. Methylphenidate does not improve cognitive function in healthy sleep-deprived young adults. J Investig Med. 2004;52(3):192-201.

12. DeVito EE, Blackwell AD, Clark L, Kent L, Dezsery AM, Turner DC, Aitken MR, et al. Methylphenidate improves response inhibition but not reflection-impulsivity in children with attention deficit hyperactivity disorder (ADHD). Psychopharmacology (Berl). 2009;202(13):531-539.

13. Agay N, Yechiam E, Carmel Z, Levkovitz Y. Non-specific effects of methylphenidate (Ritalin) on cognitive ability and decision-making of ADHD and healthy adults. Psychopharmacology (Berl). 2010;210(4):511-519.

14. Rahman S, Robbins TW, Hodges JR, Mehta MA, Nestor PJ, Clark L, Sahakian BJ. Methylphenidate ('Ritalin') can ameliorate abnormal risk-taking behavior in the frontal variant of frontotemporal dementia. Neuropsychopharmacology. 2006;31(3):651-658.

15. Kollins SH, English J, Robinson R, Hallyburton M, Chrisman AK. Reinforcing and subjective effects of methylphenidate in adults with and without attention deficit hyperactivity disorder (ADHD). Psychopharmacology (Berl). 2009;204(1):73-83.

16. Cools R, Barker RA, Sahakian BJ, Robbins TW. L-Dopa medication remediates cognitive inflexibility, but increases impulsivity in patients with Parkinson's disease. Neuropsychologia. 2003;41(11):1431-1441.

17. Sevy S, Hassoun Y, Bechara A, Yechiam E, Napolitano B, Burdick K, Delman H, et al. Emotion-based decision-making in healthy subjects: short-term effects of reducing dopamine levels. Psychopharmacology (Berl). 2006;188(2):228-235.

18. Adler LA, Spencer T, Faraone SV, Kessler RC, Howes MJ, Biederman J, Secnik K. Validity of pilot Adult ADHD Self- Report Scale (ASRS) to Rate Adult ADHD symptoms. Ann Clin Psychiatry. 2006;18(3):145-148.

19. Ward MF, Wender PH, Reimherr FW. The Wender Utah Rating Scale: an aid in the retrospective diagnosis of childhood attention deficit hyperactivity disorder. Am J Psychiatry. 1993;150(6):885-890.

20. Derogatis LR, Melisaratos N. The Brief Symptom Inventory: an introductory report. Psychol Med. 1983;13(3):595-605.

21. Volkow ND, Wang GJ, Fowler JS, Molina PE, Logan J, Gatley SJ, Gifford A, et al. Cardiovascular effects of methylphenidate in humans are associated with increases of dopamine in brain and of epinephrine in plasma. Psychopharmacology (Berl). 2003;166(3):264-270.

22. Greenberg LM, Waldman ID. Developmental normative data on the test of variables of attention (T.O.V.A.). J Child Psychol Psychiatry. 1993;34(6):1019-1030.

23. Rogers RD, Everitt BJ, Baldacchino A, Blackshaw AJ, Swainson R, Wynne K, Baker NB, et al. Dissociable deficits in the decision-making cognition of chronic amphetamine abusers, opiate abusers, patients with focal damage to prefrontal cortex, and tryptophan-depleted normal volunteers: evidence for monoaminergic mechanisms. Neuropsychopharmacology. 1999;20(4):322339.

24. Huang YS, Chao CC, Wu YY, Chen YY, Chen CK. Acute effects of methylphenidate on performance during the Test of Variables of Attention in children with attention deficit/hyperactivity disorder. Psychiatry Clin Neurosci. 2007;61(3):219-225.

25. Forbes GB. Clinical utility of the Test of Variables of Attention (TOVA) in the diagnosis of attention-deficit/ hyperactivity disorder. J Clin Psychol. 1998;54(4):461476.

26. Coons HW, Peloquin LJ, Klorman R, Bauer LO, Ryan RM, Perlmutter RA, Salzman LF. Effect of methyl- 
phenidate on young adult's vigilance and event-related potentials. Electroencephalogr Clin Neurophysiol. 1981;51(4):373-387.

27. Weyandt LL, Mitzlaff L, Thomas L. The relationship between intelligence and performance on the test of variables of attention (TOVA). J Learn Disabil. 2002;35(2):114-120.

28. Deakin J, Aitken M, Robbins T, Sahakian BJ. Risk taking during decision-making in normal volunteers changes with age. J Int Neuropsychol Soc. 2004;10(4):590-
598.

29. Turner DC, Robbins TW, Clark L, Aron AR, Dowson J, Sahakian BJ. Relative lack of cognitive effects of methylphenidate in elderly male volunteers. Psychopharmacology (Berl). 2003;168(4):455-464.

30. Arria AM, Garnier-Dykstra LM, Caldeira KM, Vincent KB, O'Grady KE, Wish ED. Persistent nonmedical use of prescription stimulants among college students: possible association with ADHD symptoms. J Atten Disord. 2011;15(5):347-356. 